ISSN: $1130-3743$

\title{
ANÁLISIS DEL ESPACIO EN LOS ENTORNOS VIRTUALES DE FORMACIÓN
}

\section{Analysis of the space in virtual training environments}

\section{Analyse de l'espace dans les environnements virtuels de formation}

\author{
Ángel García del Dujo \\ Universidad de Salamanca. Facultad de Educación. Departamento de Teoría \\ e Historia de la Educación. C/. Paseo de Canalejas, 169. 37008 Salamanca. Correo-e: \\ agd@usal.es
}

Fecha de recepción: enero de 2009

Fecha de aceptación definitiva: abril de 2009

Biblid [(1130-3743) 21, 1, 2009, 103-128]

RESUMEN

El objetivo de este artículo es analizar el carácter espacial de los entornos virtuales, es decir, ver si en los entornos virtuales está o no presente el sentido, sensación, noción de espacio; en caso positivo, se pretende, en segundo lugar, reconstruir las formas como se genera esa noción y los sentidos que adquiere en distintos entornos, es decir, identificar los mecanismos que se utilizan para generar esa noción espacial y las transformaciones conceptuales que en ese proceso se producen. En base a los resultados obtenidos se propone una interpretación pedagógica de los procesos formativos mediados tecnológicamente.

Palabras clave: espacio, lugar, entornos virtuales de formación, espacios de información, espacios de acción. 
SUMMARY

The objective of this article is to analyse the spatial character of the virtual environments, that is to say, to see whether in the virtual environments the sense, the sensation, the notion of space is present or not; in this case, it aims, secondly, to rebuild the ways this notion is generated and the senses it acquires in the different environments, that is to say, to identify the mechanisms used to generate this spatial notion and the conceptual transformations produced in this process. Based on the obtained results it is proposed a pedagogic interpretation of technologically mediated formative processes.

Key words: space, place, virtual training environments, information spaces, action spaces.

\section{SOMMAIRE}

L'objectif de cet article est analyser le caractère spatial des environnements virtuels, c'est à dire, voir si dans les environnements virtuels il est présent ou pas le sens, la sensation, la notion d'espace; dans le cas positif, il prétend, dans un deuxième lieu, reconstruire les formes comment ce notion est générée ainsi que les sens qu'elle acquiert dans les différents environnements, c'est à dire, identifier les mécanismes utilisés pour générer ce notion spatial et les transformations conceptuelles produites dans ce processus. Sur la base des résultats obtenus on propose une interprétation pédagogique des processus formatifs technologiquement entremis.

Mots clés: espace, lieu, environnements virtuels de formation, espaces d'information, espaces d'action.

\section{Aclaraciones iniciales}

Este artículo forma parte de una investigación más amplia que pretende esclarecer una de las coordenadas o supuestos inherentes a la educación no cuestionado nunca en la reflexión y en las prácticas educativas que las distintas épocas y sociedades han desarrollado y que la introducción de las nuevas tecnologías habría puesto en entredicho, al menos, habría levantado recelos y sospechas. Me estoy refiriendo al supuesto de que todo proceso de formación hace referencia implícita o explícita a una situación, es proceso situado, es decir, tiene un lugar donde acontece, que viene definido por personas implicadas y acciones que se desarrollan en alguna situación espacio-temporal; parece que esto no era motivo de discusión.

Las nuevas tecnologías habrían dado pie para cuestionar este supuesto. Por eso, la pretensión última -y el contexto general de la investigación- es estudiar si se produce ese carácter situado en los entornos virtuales de formación, si ese supuesto no cuestionado hasta ahora se sigue dando en los llamados entornos 
virtuales de formación, para lo que, concretamente, en este artículo me propongo analizar la presencia, sentidos, usos y comportamiento del espacio en los llamados entornos virtuales de formación; es un primer paso y forma de averiguar si se sigue manteniendo o no ese supuesto.

La segunda aclaración es de orden terminológico. Parece lógico acotar con precisión, ya en el punto de partida, lo que se entiende aquí por entornos virtuales de formación y, sobre todo, por espacio. Por entornos virtuales de formación entenderé los espacios generados tecnológicamente, concretamente mediante la utilización de las nuevas tecnologías de la información y comunicación, y que son susceptibles de generar, a su vez, formación. En principio no necesito acotar más este concepto; si acaso reconocer que, presentado el concepto en estos términos, habría una amplia gama de entornos, dependiendo precisamente de la tecnología utilizada. Respecto del otro término, espacio, es suficiente con tener in mente la noción más popular o coloquial en el sentido de sitio en el que se producen los acontecimientos; además, en esta investigación resulta inevitable e imprescindible hacerlo así, porque ésta es la primera noción que despierta este término cuando los profesionales lo utilizamos en educación ${ }^{1}$.

Ahora bien, es obvio que esta noción de espacio, tomada en sentido físicogeográfico, no es aplicable a los entornos virtuales de formación. Precisamente por ello y dado que está tan arraigada en el imaginario profesional de los docentes, hasta el punto de que al principio me referí a ello como supuesto no cuestionado, cuando se habla de educación en esos entornos se genera una especie de aporía que bien pudiera encontrarse en el origen de las resistencias que presenta la introducción de estas tecnologías en educación, concretamente en el caso de algunos usos y prácticas que cuestionan el concepto tradicional y casi espontáneo de espacio en educación.

Por eso, la tercera aclaración de estas reflexiones iniciales apunta, también desde el principio, al interés de esta investigación. Porque, si resulta que la web es espacial en el sentido de que el usuario tiene en todo momento un sentido y noción de espacio que concuerda con el sentido y noción usual de este término u otros conceptualmente próximos, dicho de otra manera, que el espacio está presente e interviene en la configuración de los entornos virtuales, estaríamos contribuyendo a eliminar, o al menos rebajar, una posible fuerza de resistencia para la introducción en educación de estas tecnologías, además de que serviría también en gran manera para eliminar del imaginario colectivo una idea que termina siendo perjudicial, la idea de que estas tecnologías crean un nuevo espacio, un espacio diferente al que hay que acceder para su usufructo, motivo también de sospechas

1. Espacio en el sentido de espacios, sitios, infraestructuras, escenarios de la acción. "La mayoría de los analistas sociales tratan tiempo y espacio como meros contornos de la acción y aceptan, sin advertirlo, la concepción, característica de la moderna cultura occidental, de la mensurabilidad cronológica". Giddens (2003, 143). 
y recelos por parte de los profesionales. No sería una contribución menor llegar a clarificar que en el ciberespacio, bien entendido y utilizado, no caben ni las utopías ni las distopías ni la negación de la geografía.

En algunas publicaciones anteriores (García del Dujo, 2003a, 2003b) he apuntado a las percepciones y representaciones, actitudes y creencias, que profesionales y usuarios tenemos de las propias tecnologías, no ya del modo como se produce y en qué consiste el conocimiento, como factor a considerar; es decir, variables culturales -y no epistemológicas ni metodológicas, ni siquiera propiamente pedagógicas $-^{2}$ que estarían incidiendo en la introducción y formas de uso de las nuevas tecnologías. Modos de ver e imaginar estas tecnologías derivados de una determinada concepción del espacio y tiempo y de una visión de la educación concebida en estas coordenadas y sus derivadas (el concepto de presencia como contigüidad física simultánea) que favorecerían y explicarían su uso para determinadas actividades, por ejemplo, informativas, pero no tanto para otras, más específicamente formativas.

\section{Marco teórico de la inVESTIGACióN}

Una de las principales mutaciones que está conociendo el sistema educativo es su deslocalización (Vázquez Gómez, 2002, 2003). Está en juego la forma de entender el sistema en los dos últimos siglos, la forma como las sociedades modernas estructuraron la función educativa. Desde hace un par de décadas, la llamada sociedad educativa, ciudad educadora, las otras educaciones y, en general, la interpretación de que en la sociedad de nuestros días hay muchas instancias que educan constituyen hechos y argumentos que inciden en la misma idea, la deslocalización de la educación. Junto con el otro fenómeno que inevitablemente le acompaña, la destemporalización de la educación, significan la ruptura entre lugares particulares y estadios particulares de la vida que se daba en los escenarios tradicionales (Meyrowitz, 1986).

La fase de deslocalización en la que nos encontramos, acorde por otra parte con algunas características que presenta la sociedad y la economía de nuestro tiempo (Rizvi y Lingard, 2000), implica no la desaparición de espacios pensados específicamente para la educación pero sí la negación de que lo educativo tenga lugar exclusivamente en esos espacios; sólo algunos, los más

2. A finales de la década de los noventa se publica una muy completa investigación sobre la influencia que esas variables tienen en el uso que estudiantes y docentes hacen de las nuevas tecnologías; la conclusión general fue que, sin negar su influencia, las variables de ese tipo no proporcionan una explicación totalmente satisfactoria, dando a entender que pueden estar interviniendo variables de otro tipo. Center for Research on Information Technology and Organizations (CRITO) (1998). 
radicales, hablarían de la desaparición de aquellos espacios exclusivos para la educación.

Con todo, en esta tendencia de deslocalización del fenómeno, se mantiene la noción de espacio o lugar donde se produce educación. Lo diremos mejor al revés; lo que se mantiene es el supuesto de que el proceso de formación refiere directa o indirectamente una situación, es proceso situado, es decir, tiene un lugar donde acontece definido por personas y acciones que se desarrollan en una situación espaciotemporal. Incluso, bajo un punto de vista pedagógico, los espacios donde se sitúan las acciones de formación han adquirido progresivamente una importancia notable; recordemos que detrás de las corrientes educativas que antes señalaba está la idea de proximidad, realismo, autenticidad... de escenarios, características relevantes de la pedagogía del último siglo. Más recientemente, esta perspectiva ha sido recogida por la teoría del aprendizaje situado, la cognición distribuida o las comunidades de práctica, ramificaciones psicopedagógicas de la corriente sociocultural vigotskiana, cuyo planteamiento de los procesos psicológicos superiores requiere de herramientas -materiales y simbólicas-, actividades y prácticas, todo ello instrumentos de mediación que conforman los espacios, los lugares, los contextos. En resumen, que la idea de que las acciones de formación, ya sea formal, no formal o informal, se construyen en base a relaciones entre personas en instituciones, organizaciones, o entre personas y objetos en el marco general de situaciones sociales (Waltz, 2004), ha venido siendo un supuesto no cuestionado.

El primer conflicto en este sentido habría venido de la mano de la educación a distancia y, definitivamente, lo tendríamos instalado con las nuevas tecnologías de la información y la comunicación. Estas tecnologías, para algunos, estarían cuestionando el sentido situacional, incluso el propio sentido espacial, de las acciones y procesos de formación, no ya en el sentido específico de infraestructuras materiales, que se supone, sino en el de espacialización que se atribuye a la educación. De manera que estaríamos dando un paso más allá: si hasta ahora hablábamos de deslocalización de la educación, ¿empezaríamos ahora a hablar de desmaterialización (Robert, 2004), desespacialización de la educación, convirtiendo así las acciones y procesos educativos en mero proceso informacional, representacional y computacional, incluso con riesgo de contradecir la interpretación del conocimiento como actividad social, colaborativa, participativa, no sólo perceptiva y sensorial?

Un planteamiento semejante puede verse en el análisis que hace M. Castells (1996) del espacio y el tiempo en la sociedad red, para quien los entornos virtuales de formación vendrían a ser el producto y soporte material (precisamente no espacial) de las prácticas educativas propias y características de la sociedad red, algo así como paradas momentáneas, que permiten encuentros síncronos o asíncronos, en 
el proceso constante en que consiste el espacio de los flujos que por su naturaleza, dice, trasciende y supera el espacio de los lugares ${ }^{3}$.

No extraña que algunos teóricos, sociólogos y aficionados a la ciencia ficción, coetáneos del autor que acabamos de citar, hayan terminado o creando espacios propios y característicos de esa sociedad red o simplemente hablando de interacciones sociales al margen de contextos espaciotemporales: o la imaginería del ciberespacio y las tecnópolis advenedizas (Gibson, 1994) o la negación de la geografía (Cairncross, 1997), ambos casos como resultado de una disociación creciente entre proximidad espacial y realización de las funciones de la vida cotidiana, entre la lógica del espacio de los lugares y la correspondiente al espacio de flujos, entre la experiencia y el conocimiento.

También en ambos casos la consecuencia es la misma: en palabras del propio Castells $(1998,512)$, «una esquizofrenia estructural entre dos lógicas espaciales que amenaza con romper los canales de comunicación de la sociedad" y que en el caso de la educación podría estar actuando como fuerza de resistencia al buen uso, a determinados usos, de las nuevas tecnologías entre los profesionales. No debería extrañar que los profesionales de la educación manifiesten resistencia a entrar, situarse y desarrollar su actividad en un 'escenario de flujos' que, suele añadirse además, trasciende, niega o supera las coordenadas tradicionales con las que en la vida cotidiana y profesional se vienen manejando, el espacio y el tiempo. En términos de A. Giddens (2004) estaríamos ante un segundo momento y proceso de desanclaje de la actividad social y educativa, vinculada ahora no ya a contextos naturales, locales, de presencia e inmediatez, ni siquiera a contextos institucionales y, por ende, mediatos, aunque presenciales, sino a escenarios virtuales, de no presencia.

Esto quiere decir que los mecanismos de este segundo momento y proceso de desanclaje, en la hipótesis de que se produjera, también pueden ser indagados a través de las variables de espacio y tiempo, como ya lo hiciera A. Giddens en el caso de la modernidad, si bien ahora estaríamos entendiendo dicho proceso no sólo en el sentido de desinstitucionalización sino también en el más profundo de desespacialización y hasta desmaterialización de la actividad educativa, que quedaría reducida a mero proceso de computación desprovisto de las tramas espaciales, temporales, objetuales y personales, que configuran el llamado proceso educativo.

3. "Las localidades se dislocan de sus significados culturales, históricos y geográficos, y se reintegran en redes funcionales, o en collages de imágenes, induciendo un espacio de flujos que sustituye al de los lugares. El tiempo se borra en el nuevo sistema de comunicación cuando el pasado, el presente y el futuro se puede programar para interactuar entre sí en un mismo mensaje. El espacio de flujos y el tiempo atemporal son las fundaciones materiales de una nueva cultura que trasciende e incluye la diversidad de los sistemas de representación históricamente transmitidos: la cultura de la virtualidad real, donde hacer creer es creer en el hacer». Castells (1996, 375). 
En lo que se refiere a la variable espacio, la literatura angloamericana ha resaltado su importancia para una buena y completa comprensión del funcionamiento de la Red, ya sea con una intención prioritariamente epistemológica ${ }^{4}$ (Kolb, 2000; Lankshear, Peters y Knobel, 2000, entre otros) o desde una perspectiva más sociocultural y formativa (Burbules, 2000, 2002, 2004), perspectiva en la que viene incidiendo en los últimos años la literatura pedagógica italiana (Calvani, 2001; Mantovani, 1995; Rivoltella, 2003) y en la que se inserta este trabajo, que se detiene en una cuestión previa, la sensación espacial que los usuarios tienen en los llamados entornos virtuales de formación, por considerarla de interés pedagógico.

\section{OBJETIVOS E HIPÓtesIS}

En función del planteamiento anterior, presento los objetivos concretos de este trabajo en los siguientes términos: primero, analizar el carácter espacial de los entornos virtuales, es decir, ver si en los entornos virtuales está o no presente el sentido, sensación, noción de espacio; en caso positivo, pretendo, en segundo lugar, reconstruir las formas como se genera esa noción y los sentidos que adquiere en los distintos entornos, es decir, identificar los mecanismos que se utilizan para generar esa noción espacial y las transformaciones conceptuales que en ese proceso se producen (de "espacios" a "lugares", en algunos entornos virtuales).

Expresado en términos de objetivo general, busco la deconstrucción ${ }^{5}$ del concepto de espacio en los entornos virtuales de formación por entender que este camino puede llevar a explicar por qué se produce educación en los llamados entornos virtuales de formación y a comprender cómo ese proceso de formación sigue siendo un proceso situado.

La hipótesis general y básica, donde se asienta la secuencia de objetivos que acabo de presentar, es el carácter espacial de los entornos virtuales. Explicación: la retórica y la semántica de la web son las del espacio; es éste un hecho observable en la terminología utilizada, como sustantivos, verbos y adjetivos. Ahora bien, este hecho no autoriza, apoyándose en las características de la tecnología utilizada, a pensar en la producción y conformación de un tipo o especie particular de espacio (espacio real, pero ciberespacio) ni a construir una justificación satisfactoria solamente en términos de mera metáfora del espacio físico (espacio virtual, potencial

4. "One of the most difficult challenges facing attempts to think about epistemology in relation to the Internet has to do with what we might call the Internet's spatial "ontology". Lankshear, Peters y KNOBel (2000, 17-39).

5. La deconstrucción consiste en mostrar cómo se han ido construyendo conceptos a partir de procesos históricos y acumulaciones metafóricas, mostrando que lo claro y evidente dista de serlo. La deconstrucción consiste en tomar una idea, una intuición o un valor y comprender sus mecanismos quitando el cemento que la constituye. Dicho de otro modo, la tesis que reside en esta premisa es que ninguna idea o concepto se puede transferir en forma pura. DerRIDa (1999). 
y, en consecuencia, formación virtual); ambas propuestas son manifiestamente insatisfactorias desde un punto de vista educativo, la primera por su carácter paradójico, irreal, la segunda por su connotación desespacializada, ilusionista, etérea, inmaterial, aespacial. Como decía anteriormente, en el ciberespacio, bien entendido, ni utopías ni distopías ni negación de la geografía.

Frente a estos planteamientos, la hipótesis afirma que los entornos virtuales llevan consigo una noción, sensación espacial; a su vez, esta hipótesis tiene su continuidad en esta otra: esa noción-sensación espacial, en algunos entornos, se transforma progresivamente en otros constructos conceptuales que vienen a coincidir con el significado de otro término próximo al de espacio (lugar).

\section{Estrategia y diseño METOdológico, TÉCNICAS E INSTRUMENTOS}

Para el análisis empírico del objeto de estudio con los objetivos e hipótesis señaladas, se diseñó el siguiente artificio metodológico. Dadas las dificultades para encontrar un grupo muestral donde verificar las hipótesis tal como han quedado planteadas, se optó por estudiar el comportamiento, en los aspectos que interesan a esta investigación, de un grupo de sujetos.

El grupo de sujetos ha estado formado por los alumnos del Postgrado Oficial (Máster) Las TIC en educación que se viene desarrollando en la Facultad de Educación de la Universidad de Salamanca, curso 2007-2008. Son 22 alumnos con un perfil de sexo, edad, procedencia, formación y uso de las nuevas tecnologías que se analizará en su momento. A todos ellos se les pidió que realizasen on line las siguientes actividades prácticas:

- Búsqueda de información en webs informativas (Ministerio de Educación y Ciencia y Boletín Oficial de Castilla y León) y en webs comerciales (El Corte Inglés y Media Mark).

- Participación en un foro creado con motivo de la actividad anterior en relación con las impresiones suscitadas durante la realización de la actividad, los problemas encontrados o cualquier otra valoración.

- Participación en un chat a propósito de dos artículos de Journal of Philosophy of Education. Para esta actividad el grupo se dividió en dos, de manera que se realizaron dos chats.

- Participación en una sesión en la plataforma Habbo-Hotel ${ }^{6}$, seguida del correspondiente foro.

6. Habbo-Hotel es un chat virtual on line donde los usuarios crean un personaje, pudiendo personalizarle, crear salas, realizar visitas, hacer compras, jugar... Los habbos pueden intercambiarse mensajes, saber quién se encuentra conectado en ese momento y en qué sala se encuentra, ya sea pública o privada. 
- El desarrollo de las actividades anteriores exigía la utilización de la plataforma Moodle.

Antes de continuar con la descripción del artificio metodológico utilizado, es oportuno formular y contestar una pregunta que resulta inevitable: ¿por qué estas actividades y no otras? La respuesta debe tener el mismo nivel de precisión y concisión: primero, porque son actividades diferentes ${ }^{7}$ y requieren tecnologías diferentes y, segundo, porque todas ellas, aunque diversas, quedan implicadas en el desarrollo de un buen proceso formativo, que es la actividad global que nos interesa; conviene recordar en este sentido que nuestro interés se centra en los entornos virtuales de formación.

Ahora se entenderá mejor la decisión de llevar a cabo la investigación con un grupo ad hoc, no en lo que se refiere al perfil que presenta -edad, sexo, procedencia, tipo de formación y nivel de conocimiento y uso de las tecnologíascuanto en lo relativo al tratamiento común o conjunto de experiencias que tuvieron todos sus miembros. Hubiera sido muy difícil, si no imposible, llevar a cabo esta investigación, tal como está diseñada, en términos de población-muestra, pues requiere que todos los sujetos hayan tenido las mismas experiencias en las mismas tecnologías y/o entornos.

Quedan todavía por describir algunos aspectos de lo que vengo llamando artificio metodológico que he utilizado en el estudio del objeto de investigación. Me estoy refiriendo a las técnicas e instrumentos utilizados en la recogida y tratamiento de información y análisis de datos.

Con posterioridad a la realización de las actividades reseñadas anteriormente, se aplicó un cuestionario con 42 ítems, de los que sólo trece se analizan en este trabajo, precisamente aquellos que proporcionan información relevante en la dirección de los objetivos e hipótesis señaladas, además de los seis primeros orientados a recoger información sobre el perfil de los sujetos. El tratamiento y análisis cuantitativo de los datos se ha efectuado con el paquete estadístico SPSS v.15., que realiza el análisis de la varianza a través del procedimiento Modelo Lineal General (MLG) Medidas Repetidas ${ }^{8}$.

7. El elenco de actividades implicadas va desde la búsqueda de información, ya sea en web meramente informativa (MEC y BOCyL) o comercial (Corte Inglés y Media Mark), hasta la comunicación, interacción y realización de acciones en entornos virtuales gráficos que reproducen escenarios de la vida real (Habbo-Hotel), pasando por la participación escrita en espacios virtuales síncronos (Chat) y asíncronos (Foro). Y todo ello utilizando una plataforma de enseñanza-aprendizaje (Moodle).

8. "(que)... proporciona un análisis de varianza cuando se toma la misma medida varias veces a cada sujeto o caso. Si se especifican factores inter-sujetos, éstos dividen la población en grupos. Utilizando este procedimiento de modelo lineal general, puede contrastar hipótesis nulas sobre los efectos tanto de los factores inter-sujetos como de los factores intra-sujetos. Asimismo puede investigar las interacciones entre los factores y también los efectos individuales de los factores. También se pueden incluir los efectos de covariables constantes y de las interacciones de las covariables con los factores inter-sujetos". Pérez López (2005, 359). 


\section{Resultados}

\subsection{Caracterización de los sujetos}

Las variables utilizadas para definir el perfil de los sujetos han sido las siguientes: sexo, edad, procedencia, tipo y nivel de formación, nivel de conocimiento y uso de algunos programas de ordenador y de algunas actividades en Internet. Dado el escaso número de sujetos, se procedió en algunas variables a una recodificación para evitar la dispersión. El perfil obtenido responde a las siguientes características:

\author{
Hombre $=36,4 \%$ \\ Entre 20 y 30 años $=86,4 \%$ \\ Españoles $=54,5 \%$ \\ Estudios superiores en educación $=59,1 \%$
}

\author{
Mujer $=63,6 \%$ \\ Más de 30 años $=13,6 \%$ \\ Extranjeros $=45,5 \%$ \\ En otras titulaciones $=40,9 \%$
}

Con un conocimiento y uso avanzados de herramientas o programas de ordenador, que supera el $75 \%$ en el caso de programas habituales -procesador de texto $(77,3 \%)$, reproductor de música $(86,4)-$, mientras que muestran un nivel básico en el caso de programas más específicos -lenguajes de programación (4,8\%), creación de páginas web (18,2\%)-. Y con un buen conocimiento y uso de actividades relativas al envío y recepción de mensajes -comunicación email (100\% en nivel avanzado)-, búsqueda de información en web (86,4\%), comunicación en chat (77,3\% en nivel avanzado); un conocimiento medio en actividades de enseñanzaaprendizaje $(59,1 \%)$, compras on line $(47,6 \%)$, intervención en foros $(45,5 \%)$ y un nivel básico en búsqueda de información en bases de datos (40,9\%), operaciones financieras on line $(36,4 \%)$, juegos on line $(27,3 \%)$, publicación en blogs $(27,3 \%)$ y juegos en MUD (13,6\%).

En comparación con el perfil de usuario proporcionado por otros estudios, podemos decir que se trata de sujetos que se ajustan al perfil de usuario habitual, ya sea hombre o mujer, en cuanto a conocimiento y uso de actividades en Internet y también en cuanto a conocimiento y uso de programas de ordenador?.

\subsection{Comprobación de hipótesis}

La primera hipótesis afirma el carácter espacial de la web. A la vista de los datos resultantes del tratamiento estadístico al que hemos sometido la información

9. En adelante, de estas dos variables -nivel de conocimiento y uso de algunos programas de ordenador y algunas actividades en Internet- tomaremos como referencia las relativas al conocimiento y uso de actividades de enseñanza-aprendizaje y compras on line. 
proporcionada por los ítems analizados, podemos concluir que los sujetos del grupo de estudio han experienciado la web (los distintos entornos presentados) en sentido espacial. En el conjunto del grupo de estudio y para cada una de las tecnologías señaladas hay altos porcentajes de sujetos que tienen esta sensación (ya sea como espacio o como lugar) ${ }^{10}$. La media de sensación de espacio (en una escala de 0 a 100) oscila entre el $42,85 \%$ en el caso del foro y el $74,76 \%$ en la web comercial (la sensación de lugar lo hace entre el 27,38\% de la web comercial y el 81,36\% del chat), con una desviación típica que, en general, está indicando un buen grado de homogeneidad en la respuesta. No se aprecian diferencias significativas en relación con las variables sexo, edad, nivel de formación, procedencia, conocimiento y uso de plataformas de enseñanza-aprendizaje y conocimiento y uso de compras on line.

TABla 1. Sensación de espacio/lugar por tecnología ${ }^{11}$

\begin{tabular}{|l|c|c|c|c|}
\hline \multirow{2}{*}{} & \multicolumn{2}{|c|}{ Espacio } & \multicolumn{2}{c|}{ Lugar } \\
\cline { 2 - 5 } & $\bar{X}$ & $\mathrm{~S}_{\mathrm{X}}$ & $\bar{X}$ & $\mathrm{~S}_{\mathrm{X}}$ \\
\hline Web informativa & 69,76 & 24,31 & 29,52 & 25,78 \\
\hline Web comercial & 74,76 & 23,95 & 27,38 & 26,05 \\
\hline Chat & 45,77 & 31,72 & 81,36 & 15,74 \\
\hline Foro & 42,85 & 27,99 & 68,86 & 22,62 \\
\hline Habbo-Hotel & 60,00 & 35,55 & 44,90 & 37,25 \\
\hline Moodle & 47,50 & 30,18 & 73,18 & 19,18 \\
\hline
\end{tabular}

La información proporcionada por otros varios ítems confirma, al tiempo que matiza y complementa, lo que acabamos de afirmar. Cuando se pregunta a los sujetos por la experiencia que han tenido, en los distintos entornos virtuales, respecto de un conjunto de sensaciones que en los escenarios tradicionales ayudan a conformar la sensación espacial -sensación de realidad, de orientación, de entrar y salir, de movimiento, de acción y control de la situación-, no se constata que, durante la realización de las actividades, haya habido un fuerte grado de desorientación, confusión, pérdida de sensación de realidad o deterioro de esos otros

10. Todos los autores consultados, pero preferentemente arquitectos, antropólogos y sociólogos, y por este orden, entienden que el concepto de lugar supone el concepto de espacio.

11. Ítem 42. Si tu sensación de espacio en el mundo real es del 100\% y tu sensación de lugar, en aquellos espacios que para ti son lugares, es también del 100\%, ¿en cuánto estimarías tu sensación de espacio y de lugar para cada una de las actividades realizadas? (utiliza una escala del 0 al 100). Este ítem ha sido sometido a un doble tratamiento estadístico: descriptiva general y prueba de t para muestras independientes, que permite comparar las medias del ítem en los dos grupos definidos (espacio y lugar) respecto de las distintas variables independientes consideradas. 
componentes o mecanismos que configuran la sensación espacial; el análisis de varianza de medidas repetidas viene a confirmarlo.

Eso sí, hemos comprobado que, al menos en algunos ítems, las sensaciones correspondientes no son del mismo tipo ni intensidad, por ejemplo, la sensación de movimiento es menor en el caso de las webs informativa y comercial; en algunos casos hemos visto diferencias significativas entre algunas de las tecnologías o entornos utilizados, con indicios de llegar a presentar una especie de polarización entre ellas, pero estamos entrando en la hipótesis siguiente.

La segunda hipótesis afirma precisamente que en los entornos virtuales esa sensación presenta-evoluciona-se transforma en distintos tipos, formatos, órdenes de espacialidad, dando paso en algunos casos a una sensación espacial más "evolucionada"; dicho de otra manera, en algunos entornos la sensación de espacio se transforma progresivamente en otros constructos experienciales-conceptuales que vienen a coincidir con el significado de otro término próximo (lugar), que también conforma el convoy semántico espacial.

Recuperando los datos de la tabla anterior, se observa que el orden que presentan los distintos entornos en relación con la sensación de espacio (en la tabla siguiente en orden decreciente) es, en los tres primeros casos, el inverso que presentan esos mismos entornos en relación con la sensación de lugar (en la tabla siguiente en orden creciente). Los sujetos de estudio estarían formando dos bloques con los entornos utilizados: uno con predominancia de sensación de espacio, otro de lugar; el primero en base a la web, comercial e informativa, el segundo en torno al chat y Moodle.

TABla 2. Sensación de espacio/lugar por tecnología, en orden cruzado

\begin{tabular}{|l|c|c|c|c|l|}
\hline \multirow{2}{*}{} & \multicolumn{2}{|c|}{ Espacio } & \multicolumn{2}{c|}{ Lugar } & \multirow{2}{*}{} \\
\cline { 2 - 5 } & $\bar{X}$ & $\mathrm{~S}_{\mathrm{X}}$ & $\bar{X}$ & $\mathrm{~S}_{\mathrm{X}}$ & \\
\hline Web comercial & 74,76 & 23,95 & 27,38 & 26,05 & Web comercial \\
\hline Web informativa & 69,76 & 24,31 & 29,52 & 25,78 & Web informativa \\
\hline Habbo-Hotel & 60,00 & 35,55 & 44,90 & 37,25 & Habbo-Hotel \\
\hline Moodle & 47,50 & 30,18 & 68,86 & 22,62 & Foro \\
\hline Chat & 45,77 & 31,72 & 73,18 & 19,18 & Moodle \\
\hline Foro & 42,85 & 30,18 & 81,36 & 15,74 & Chat \\
\hline
\end{tabular}

La trayectoria y diferencias en cada caso se aprecian perfectamente en la representación gráfica siguiente, que muestra cómo los sujetos del grupo de estudio han experienciado los entornos virtuales utilizados en dos formatos/órdenes de espacialidad: unos como mero espacio, otros como lugares. 
GRÁFICO 1

Representación de medias de sensación de espacio y de lugar por tecnologías

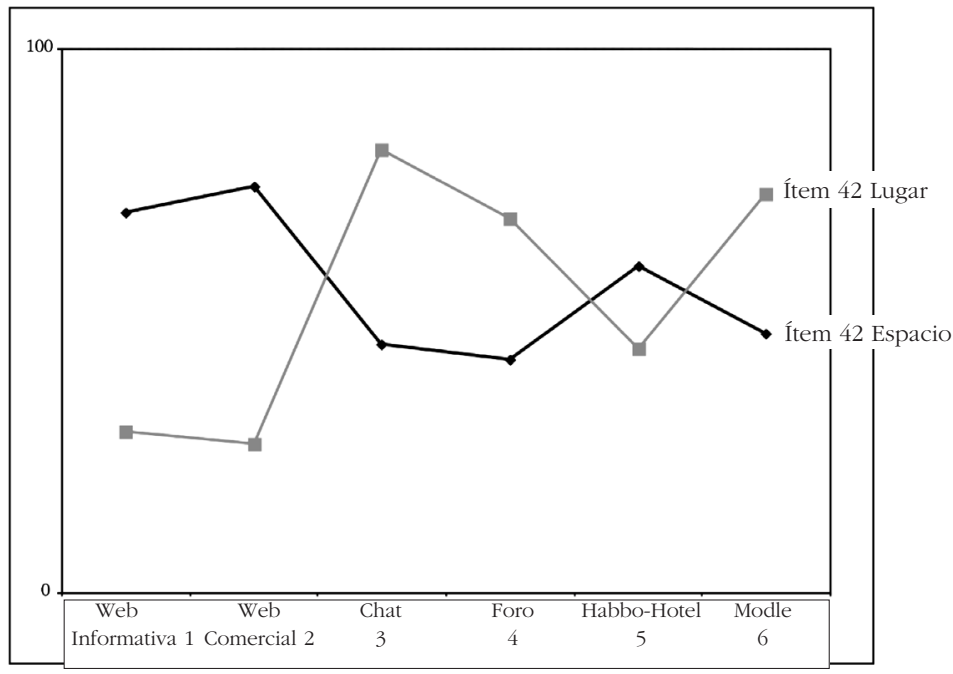

Y como en la hipótesis anterior, también en este caso la información de otros ítems confirma, matiza y complementa lo que acabamos de afirmar. Veamos.

Algunos de esos ítems buscaban información acerca de la imagen que los sujetos generan sobre sí mismos, durante la realización de las actividades, sobre dónde están y qué están haciendo. Después del tratamiento estadístico correspondiente, se comprueba que los sujetos han formado con los entornos utilizados dos bloques bien diferenciados: uno, en el que su autoimagen es la de un sujeto que ve cosas, visita sitios (con una sensación simplemente indiferente respecto de estar en el espacio o fuera del entorno); otro, en el que esa imagen es bien diferente, pues el sujeto se ve como haciendo cosas en el interior de ese sitio; el primero formado por las web comercial e informativa, el segundo por el chat y Moodle. El primero bien puede considerarse un espacio de información, el segundo un espacio de acción. Este segundo bloque presenta indicios de ser para ellos algo más que mero espacio, un lugar para ellos, se ha transformado de espacio en lugar. La representación gráfica así lo manifiesta. 
GrÁFICO 2. Representación gráfica de la media de cada tecnología ${ }^{12}$

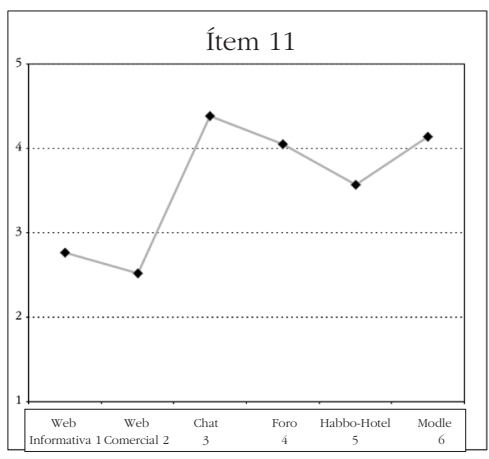

GrÁFICO 3. Representación gráfica de la media de cada tecnología ${ }^{13}$

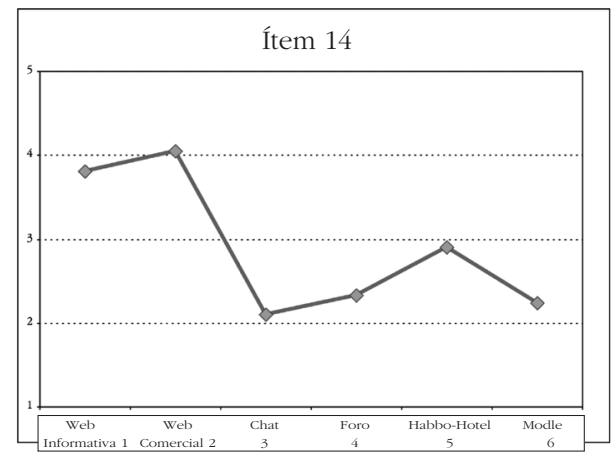

En ambos casos el nivel crítico (de significación) es menor que 0,05, concluyendo que hay diferencias significativas respecto de las distintas tecnologías. Dichas diferencias se observan precisamente en el espacio 3 (chat) respecto del 2 (web comercial), y en el 6 (Moodle) respecto del 5 (Habbo-Hotel), sin apreciarse diferencias significativas en relación con las variables independientes -sexo, edad, formación, procedencia y conocimiento- y uso de actividades en Internet -enseñanza/aprendizaje en una plataforma y compras on line-.

Otros ítems iban dirigidos a obtener información sobre los componentes, mecanismos y procesos que intervienen en la transformación de los espacios en lugares. $\mathrm{Y}$ en este sentido se comprueba que en esa transformación de espacio a lugar que los sujetos atribuyen a algunos de los entornos virtuales utilizados intervienen componentes y mecanismos análogos a los que utilizamos en los escenarios tradicionales -entre otros, la realización de acciones que dejan huella e incitan al regreso a ese lugar, emociones positivas y ambiente de compañerismo y cooperación, sensación progresiva de pertenencia a un grupo en cuyo interior se entrelazan esas acciones y emociones-.

Como mejor se aprecian los resultados encontrados en este punto es a través de la representación gráfica. En esta ocasión, presento sólo los gráficos de algunos de los muchos ítems aquí utilizados y tratados estadísticamente. En todos ellos puede verse que la respuesta de los sujetos al factor al que se refiere la

12. Ítem 11. Durante la realización de las actividades tuve más sensación de estar y de actuar en un espacio que de estar viendo y manejando algo desde fuera. Este ítem, y los siguientes, han sido sometidos a un doble tratamiento estadístico: descriptiva general y medidas repetidas.

13. Ítem 14. Durante la realización de las actividades tuve más sensación de ver cosas que de hacer cosas. 
pregunta es siempre más favorable en los casos del chat, Moodle y foro y mucho más desfavorable en el caso de la web comercial y de la web informativa. Llama la atención en todos los casos la alta representatividad que tiene la media en el caso del chat, lo mismo que le ocurre a la web comercial e informativa, pero en sentido contrario. Conviene recordar que la escala de valoración utilizada es la siguiente: 1 = en total desacuerdo; 2 = en desacuerdo; 3 = indiferente; 4 = de acuerdo $\mathrm{y}$ 5 = muy de acuerdo.

En lo que se refiere al factor emocional, donde los sujetos se sintieron más involucrados en sentido positivo fue en el chat y en Moodle y en sentido negativo en las webs informativa y comercial, observándose en ambos casos diferencias significativas en relación con el sexo y la edad.

GRÁFICO 4. Representación gráfica de la media de cada tecnología ${ }^{14}$

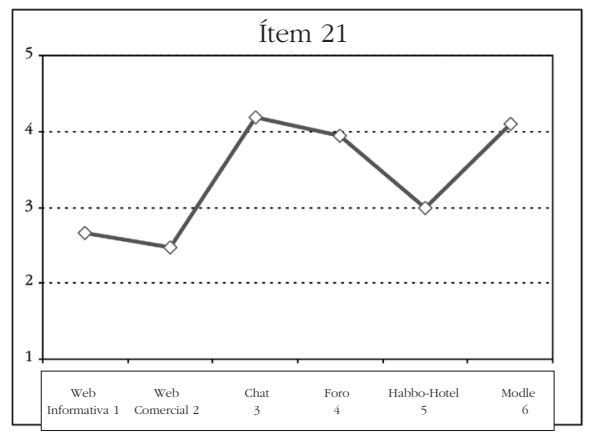

GráfICO 5. Representación gráfica de la media de cada tecnología ${ }^{15}$

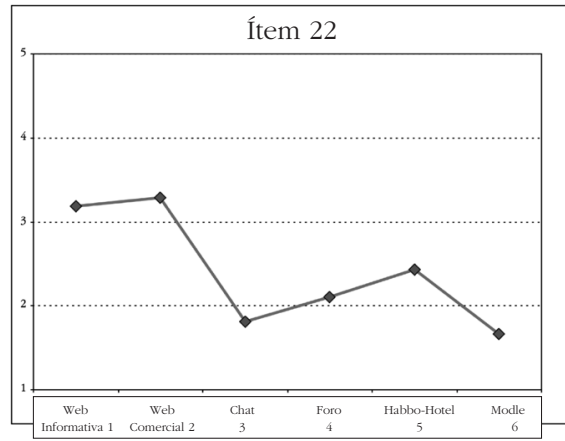

Algo parecido ocurre en lo que se refiere a acciones significativas que dejan huella e incitan al regreso.

14. Ítem 21. Durante la realización de las actividades me sentí emocionalmente involucrado en sentido positivo: muy a gusto, confortable, satisfecho.

15. Ítem 22. Durante la realización de las actividades me sentí emocionalmente involucrado en sentido negativo: desbordado, con un cierto malestar y frustración. 
GRÁFICO 6. Representación gráfica de la media de cada tecnología ${ }^{16}$

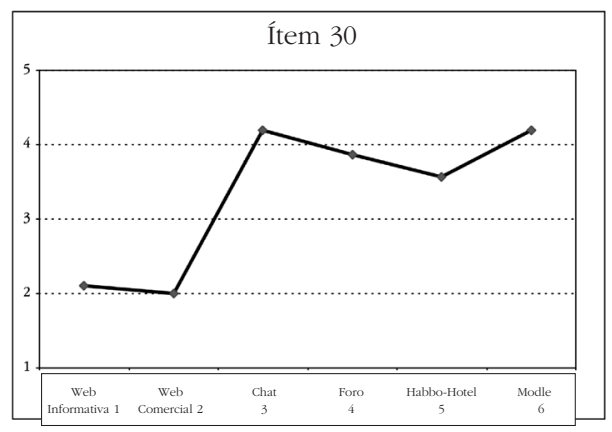

GráfICO 7. Representación gráfica de la media de cada tecnología ${ }^{17}$

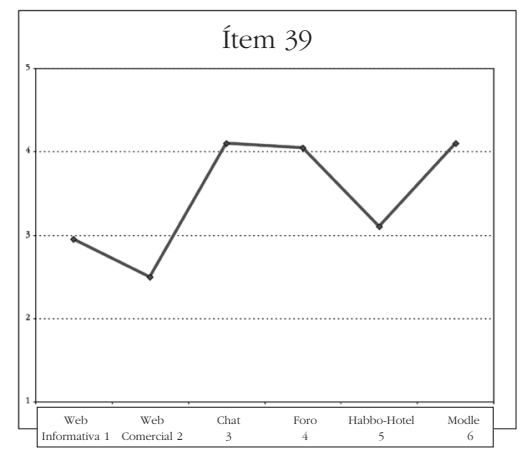

Y también respecto de la formación de un ambiente de compañerismo, cooperación y sensación progresiva de pertenencia a un grupo.

GRÁFICO 8. Representación gráfica de la media de cada tecnología ${ }^{18}$

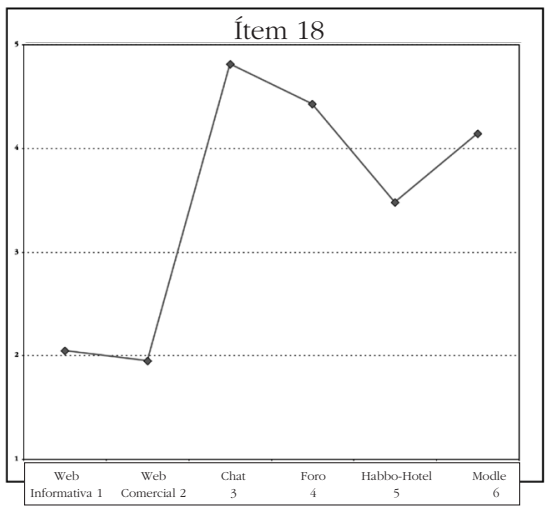

GrÁFICO 9. Representación gráfica de la media de cada tecnología ${ }^{19}$

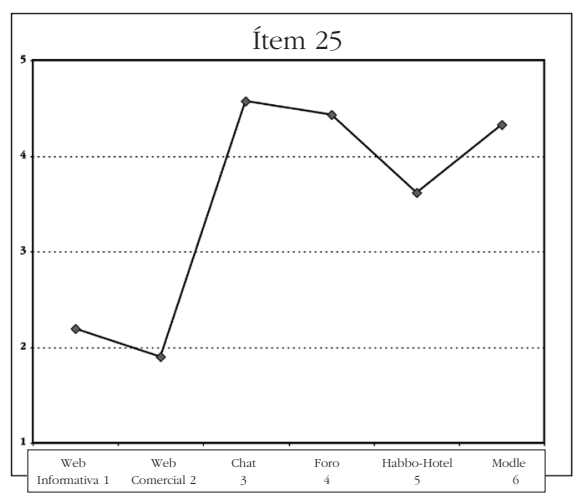

16. Ítem 30. Durante la realización de las actividades me daba cuenta de que mis acciones producían cambios en los sitios donde estaba.

17. Ítem 39. Cuando pienso en las actividades desarrolladas me vienen ganas de haber estado más tiempo, incluso de volver a entrar en algunos sitios.

18. Ítem 18. Durante la realización de las actividades tenía la sensación de que estaba haciendo cosas con mis compañeros.

19. Ítem 25. Durante la realización de las actividades fui teniendo la sensación de que formaba parte de un grupo. 
Así como respecto de la interdependencia de acciones, opiniones y sensaciones.

GRÁFICO 10. Representación gráfica de la media de cada tecnología ${ }^{20}$

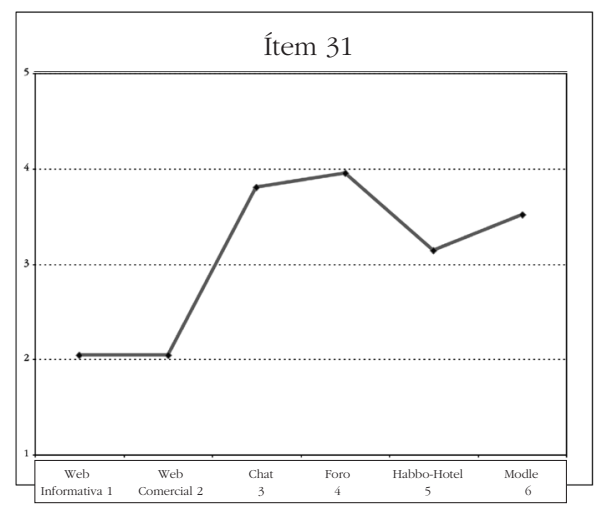

GRÁFICO 11. Representación gráfica de la media de cada tecnología ${ }^{21}$

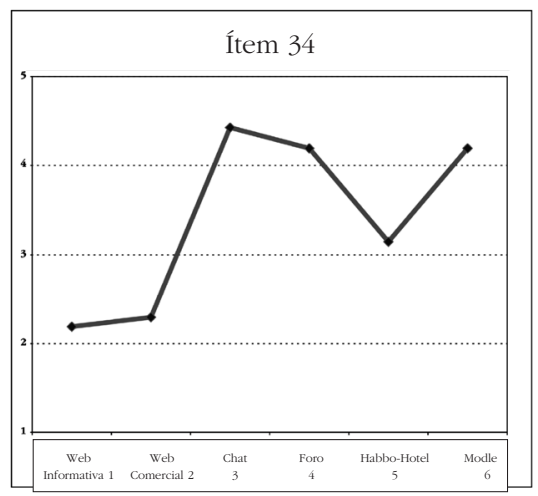

A la vista de los resultados anteriores cabe pensar que en los entornos virtuales la forma de generar la noción espacial y de transformar esos espacios en lugares varía en función de la tecnología utilizada y del tipo de actividad que se pretende implementar en ellos. Dicho de otra manera, la forma como las nuevas tecnologías "juegan" con el espacio varía con el tipo de uso que hacemos de ellas, que a su vez se relaciona, lógicamente, con la tecnología utilizada.

Es la actividad, lo que se puede hacer y lo que se hace en los sitios, el nivel, tipo y forma de llevar a cabo la actividad que los sujetos despliegan, la que consigue no sólo generar la sensación espacial sino transformar algunos espacios en lugares ${ }^{22}$. Una cosa es la sensación de movimiento, de entrar y salir, que se requiere para que haya sensación de espacio y otra bien diferente es la acción concreta que el sujeto pone en marcha y la forma como lo hace.

El elenco de actividades con el que hemos trabajado va desde la búsqueda de información en tecnologías ad hoc (web informativa o comercial, puesto que en estas últimas no se podía realizar transacciones mercantiles) hasta la participación

20. Ítem 31. Durante la realización de las actividades mis acciones eran dependientes de las acciones de los otros y viceversa.

21. Ítem 34. Durante la realización de las actividades me daba cuenta de que mis acciones, opiniones y sensaciones se entrelazaban con las de mis compañeros.

22. Conviene recordar que en su momento -ver nota 10- justifiqué el elenco de actividades elegidas por ser diferentes, por requerir tecnologías diferentes y porque todas ellas quedan implicadas en el desarrollo de un buen proceso formativo, que es la actividad global que nos interesa. 
en chats o la comunicación e interacción en Habbo-Hotel. Y, según muestran los gráficos, mientras unas actividades (permitidas por las correspondientes tecnologías, por supuesto) generan en los sujetos una fuerte sensación de estar metidos en ellas, otras generan la sensación contraria, como si los sujetos estuvieran fuera de la propia actividad.

GRÁFICO 12. Representación gráfica de la media de cada tecnología 23
GRÁFICO 13. Representación gráfica de la media de cada tecnología ${ }^{24}$
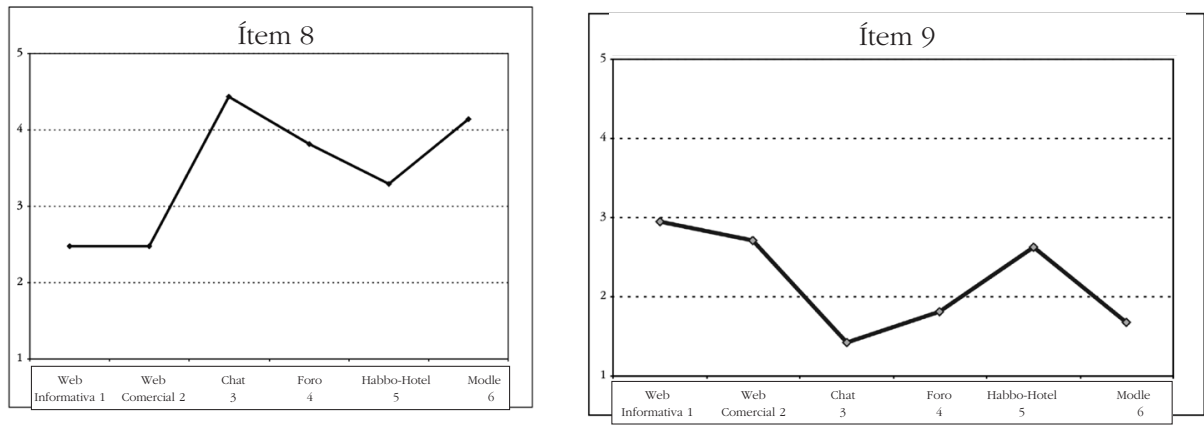

6. INTERPRETACIÓN Y DISCUSIÓN PEDAGÓGICA: DECONSTRUCCIÓN DEL ESPACIO EN LOS ENTORNOS VIRTUALES DE FORMACIÓN

Del planteamiento y desarrollo de la investigación, así como de los resultados obtenidos, me interesa recuperar en este momento lo siguiente: la sensación de espacio/lugar que los sujetos sometidos a estudio tuvieron de los seis entornos tecnológicos, generándose claramente dos bloques - uno con predominio de sensación de espacio, otro con predominio de sensación de lugar- que acogen actividades diferentes: en el primer caso, de tipo informativo, en el segundo más bien participativo, interactivo ${ }^{25}$.

23. Ítem 8. Durante la realización de las actividades tuve una fuerte sensación de estar ahí.

24. Ítem 9. Durante la realización de las actividades parecía como si yo estuviera fuera de la propia actividad.

25. Recordaré también que éste era el objetivo de este artículo, cuyos datos pertenecen a una investigación cuya pretensión última es analizar el carácter situado de los procesos formativos mediados tecnológicamente, lo que, a su vez, dije al inicio del trabajo, podría ayudarnos a entender el comportamiento bifronte de los profesionales en función del tipo de uso que se hace de las nuevas tecnologías: una actitud o comportamiento favorable, cuando se trata de acceder, enviar, recibir información (también en el caso de la comunicación cuando se entiende en términos de 
En educación estas tecnologías nos interesan en los dos sentidos, como espacios de información y, sobre todo, como generadoras de espacios de acción, llamados también lugares. Es la doble concepción de estas tecnologías que ya avanzara J. Weston (1994) y que puede verse en otros muchos autores:

It is certainly inadequate to view "it" (simply) in terms of an information infrastructure involving multiple discrete but connectable 'technologies'- Neither is it appropriate to think of the Internet in terms solely of information and data except, perhaps, in some trivial sense in which anything that is communicated can sooner or later be called data or information. Instead, we can envisage the Internet as a range of technologically-mediated spaces of communicative practice that are amazingly diverse -a multiplicity of language games that are by no means confined to informing, and that are not best understood solely in terms of content (Lankshear, Peters y Knobel, 2000).

siendo la primera incluso de importancia secundaria, en alusión explícita al enfoque de J. Weston;

Hence, if we are to understand the Internet in more than merely infrastructural and technicist terms, or as a massive conduit for information transmission, retrieval and manipulation -which we must- we need understand the ways in which the relational aspects of the diverse kinds of practices and purposes played out there "qualify and define what gets transmitted as content".

Es la concepción, en fin, que se corresponde con la distinción entre "space" y "place", distinción de vital importancia para el planteamiento desarrollado en esta investigación.

Pues bien, ¿cómo 'leer' los resultados obtenidos en perspectiva pedagógica? ¿Qué función y sentido tiene esa sensación de espacio/lugar que los sujetos han manifestado en los dos bloques de entornos utilizados?

En el primer caso, actividades de tipo informativo, de búsqueda de información -incluso en las de comunicación cuando queda reducida a transmisión de información- está interviniendo un concepto y sentido de espacio que se concreta y materializa en el punto de salida, punto de llegada y trayecto o distancia a recorrer entre el uno y el otro. Las nuevas tecnologías enfatizan aquí dos aspectos clásicos: la idea de transferencia de información y la de velocidad, rapidez en el traslado o transporte de algo de un punto a otro, ya sea el propio sujeto o algo interpuesto quien porte, lleve, dé traslado al contenido de la información. De manera que es el sitio de partida, el de llegada y el trayecto a recorrer quienes adquieren relevancia; en todo caso el énfasis se está poniendo en la infraestructura.

simple transmisión de información) y una actitud diferente (recelosa) cuando la actividad deja de ser meramente informativa/comunicativa y pasa a ser propiamente formativa. 
Estos conceptos - de espacio, de información y de comunicación como transmisión de información-, así entendidos, conducen a una imagen o representación de las nuevas tecnologías como instrumentos para enviar o recibir información con independencia del sitio donde se encuentre la información y/o el sujeto receptor, así como de las características del trayecto o espacio a recorrer ${ }^{26}$.

Dicha concepción juega fundamentalmente con la velocidad en la transferencia de información y, en el caso de las nuevas tecnologías, conduce a una sensación de compresión del espacio y el tiempo, resaltada por muchos autores (Echeverría, 1999), pero no altera las coordenadas espaciotemporales en las que se desarrolla la actividad concreta del usuario; más aún, los movimientos que lleva a cabo en la localización/rastreo, etc., del sitio o entorno donde se encuentra la información -espacio informacional- le generan una sensación espacial, según hemos visto en el marco de la primera hipótesis. Un ejemplo clarificará lo que estoy tratando de decir: en la presentación de la información que hace el profesor en el aula alude con frecuencia a otros documentos, artículos, libros, planteamientos de otros autores... En esa situación el profesor no tiene inconveniente en acercarse a un ordenador, conectado a Internet, para acceder a esa información, porque esta acción no altera las coordenadas espaciotemporales de su actividad formativa; además, en el acceso y movimiento por esa tecnología encuentra sensación espacial.

Por lo tanto, el profesional, vencidas las primeras resistencias por falta de habilidad técnica para el manejo del instrumento o aparato, no tiene inconveniente en usar esos artefactos como herramientas en el mismo sentido que siempre ha utilizado otras herramientas en el aula. La concepción se soporta aquí sobre la idea de transferencia de información de un sitio a otro y se acepta de buen grado el hecho de que se utilicen instrumentos o medios cada vez más rápidos ${ }^{27}$. Detrás de este planteamiento se encuentra, obviamente, la teoría matemática de la comunicación de Shannon y Weaver y la imagen del cerebro como máquina de cómputo.

Por otra parte, esta imagen o representación de las nuevas tecnologías en cuanto herramientas consiente una concepción de la educación y del conocimiento en términos de transmisión de información, simple y mera instrucción, muy extendida entre los profesionales de la enseñanza. Pero, incluso en aquellos

26. La representación gráfica correspondiente sería la de un sujeto delante de una pantalla, buscando, viendo, mirando, visitando sitios, espacios de información, que coincide con la autoimagen que los sujetos de este estudio tienen en la actividad de búsqueda de información en web, ya fuese informativa o comercial.

27. La imagen predominante de las nuevas tecnologías es, entre los no usuarios, como herramientas de comunicación y entre los usuarios, como herramientas de información. Ésta es la concepción predominante en los discursos de acompañamiento a estas tecnologías, tanto en la literatura científica como no científica, así como en la de consumo. Investigación realizada con sujetos entre 11 y 17 años con participación de España, Canadá, Francia, Italia, Bélgica... Puede verse en http://cepad. unicatt.it/ragazziweb/. En ambos casos, la concepción se soporta sobre transferencia de información de un sitio a otro. 
profesionales que entienden el proceso de enseñanza-aprendizaje en términos más constructivistas, esta representación no interfiere en dicha concepción; al contrario, el acceso y presentación de información, como sabemos desde hace tiempo, es una de las dimensiones del proceso de enseñanza-aprendizaje entendido "more constructivista".

Por último, esta concepción que pivota sobre la transferencia de información de un sitio a otro conduce a una interpretación de la Red como gran contenedor o almacén, conectando con uno de los sentidos en que se ha conceptualizado el espacio a lo largo de la historia, un sentido objetivista: así se explica para muchos autores el predominio de metáforas geográficas.

Pero no parece que esta descripción sea suficiente para explicar cómo se produce un proceso de enseñanza-aprendizaje mediado por estas tecnologías. Cuando nos encontramos en un entorno virtual de aprendizaje, no es ésa la noción de espacio ni de comunicación que estamos manejando, tampoco lo es la concepción de las nuevas tecnologías como meros artefactos primarios.

Dicho de otra manera, por esta vía encontramos explicación satisfactoria para la relación entre hombre-máquina y su uso en actividades educativas, sin forzar ni alterar la concepción primaria que el sujeto tiene tanto de los aparatos -instrumentos para hacer cosas- como de las prácticas educativas, en términos de prácticas sociales compartidas en el tiempo, consistentes también en transmisión de información de unas personas a otras, lo que se ha asimilado tradicionalmente a la noción de contigüidad. Pero esta explicación es insuficiente para dar razón de la relación total que se establece entre persona y persona mediada tecnológicamente (persona-máquina-persona), relación cuya comprensión resulta imprescindible para entender adecuadamente cómo puede producirse actividad formativa mediante las nuevas tecnologías.

Los conceptos de comunicación, espacio y artefactos primarios, tal como se entendían allí, resultan ahora inadecuados. Se requiere que las nuevas tecnologías sean vistas de otra manera, lo que a su vez conlleva e implica otros sentidos de comunicación y de espacio. La ausencia de estas "segundas concepciones" es lo que explicaría la actitud resistente de unos y otros a utilizar las nuevas tecnologías con propósito plenamente formativo. Ello no quiere decir que esas "primeras nociones" de comunicación, espacio y artefactos primarios desaparezcan, pero sí se necesita que se reconfiguren conceptualmente, arrastrando consigo una reconfiguración conceptual de los componentes teóricos y prácticos de la actividad educativa: el concepto de comunicación ha de acoger también la idea de acción/interacción, el concepto de espacio ha de dar paso al sentido de lugar o situación en la que se hacen cosas. El concepto de artefactos primarios ha de ser completado con la idea de artefactos secundarios o instrumentos que no solamente permiten hacer cosas sino que técnicamente son capaces de generar espacios donde se pueden hacer cosas, es decir, escenarios de acción (Pearson y Somekh, 2003).

Conviene reparar en los nuevos términos que estoy utilizando para construir una explicación coherente de la actividad formativa mediada por estas tecnologías 
y que, en definitiva, son dos, aunque podemos utilizar varias denominaciones: entornos de acción, espacios de acción, escenarios de acción (places, non spaces); obviamente se trata de espacios de información (no geográficos ni físicos ni matemáticos) que adquieren la categoría de entornos o escenarios porque permiten la acción e interacción en base o alrededor de la información.

Las ideas importantes también son dos: que en esos entornos se pueden hacer cosas (acciones) y que ello es visible para los demás, lo que les convierte en espacios inherentemente sociales. En este sentido podríamos decir que esos entornos virtuales "están habitados", despliegan socialidad. El siguiente texto recoge este planteamiento:

... a set of Web pages does not constitute a virtual learning environment unless there is social interaction about or around the information. This includes synchronous (e.g. chat, MUDs...) versus asynchronous (e.g. electronic mail, forums), communication, one-to-one versus one-to-many or many-to-many, text-based versus audio and video... This includes also indirect communication such as sharing objects.

What is specific to virtual environments compared to an information space is that it is populated. The users are inside the information space and see a representation of themselves and/or others in the space. As soon as students see who else is interested by which information, the space becomes inherently social ${ }^{28}$.

Sigue diciendo, recogiendo a su vez el pensamiento de otros autores, que los investigadores han introducido la noción de place para enfatizar el carácter social de ese espacio, es decir, la noción de espacio social. En terminología de otros autores, son los llamados lugares sociales.

While spaces take their sense from configuration of brick, mortar, Word and glass, places take their sense from configurations of social actions. Places provide what we call appropriate behavioural framing (Dourish, 1999).

En resumen, que acción y visibilidad, y en consecuencia interacción, son los rasgos distintivos que transforman los espacios de información en entornos virtuales de aprendizaje. La potencialidad formativa de estas tecnologías se explicaría porque técnicamente son capaces de crear un médium (la idea de escenarios, espacios) que hace posible la coexistencia y combinación de acciones

28. Dillenbourg (2000). Destacaré aquí la coincidencia de la descripción que hace el autor de los entornos virtuales con las respuestas que el grupo de sujetos estudiados dieron a aquellos ítems de la hipótesis segunda que preguntaban por su imagen en relación con el chat, Moodle, Foro e incluso Habbo-Hotel. Recordemos que se veían a sí mismos dentro del espacio de acción, realizando acciones en el mismo sitio que sus compañeros, etc. 
y situaciones de distinto tipo, procedencia y nivel ${ }^{29}$, sin requerir contigüidades espaciales o temporales, lo que no significa negar la existencia de componentes y entramados espaciales y temporales.

Algunos aspectos de estas afirmaciones necesitan ser ampliados, por supuesto, pero son suficientes para dejar claro que el énfasis aquí no se pone en el hecho de hacer compatible la simultaneidad con la distancia, es decir, en aquellos aspectos más próximos a la teoría matemática de la información sino en la acción y en la situación, aspectos que abren las puertas a una reconfiguración teórica del proceso de comunicación y de la educación más acorde con los planteamientos actuales, una forma de explicar las prácticas y usos de las nuevas tecnologías, concretamente las prácticas y usos educativos, en términos de la teoría sociocultural y/o alguna de sus derivadas, por ejemplo, la teoría de la acción situada.

En resumen, toda una reconfiguración semántica del convoy terminológico que gira en torno a la educación mediada tecnológicamente y que arranca y se sostiene en la reconfiguración conceptual que experimenta el término espacio: hablo de reconfiguración conceptual, no de negación ni supresión del espacio. Ésta era, y hasta aquí llegaba, precisamente la pretensión de este artículo. Son la acción y el sistema de acciones e interacciones que se despliegan alrededor de la información los que generan el sentido espacial de los entornos virtuales de aprendizaje, que poco a poco van adquiriendo el atributo de lugares como resultado de la intervención de factores y mecanismos, por cierto no muy diferentes de los que intervienen en contextos presenciales.

\section{CONCLUSIÓN}

Los resultados que aquí presento son congruentes con los que vengo obteniendo en trabajos anteriores, al tiempo que representan un paso adelante. En otros trabajos, he señalado la necesidad de diferenciar, cuando hablamos de formación on line, entre entornos de información y de comunicación y entornos de formación; también he insistido en la necesidad de poner de manifiesto la teoría pedagógica que subyace a los procesos educativos on line, incluso he afirmado que la perspectiva más apropiada para ello debería venir de la mano de la corriente sociocultural y/o sus derivadas. Dicho en otras palabras, procede superar ya la fase administrativa, política, ingenieril, en la que muchas veces da la impresión de que se encuentra la investigación sobre nuevas tecnologías en educación, hablando de infraestructuras, tiempos y tasa de ordenadores por centro y alumno, para adentrarnos en los usos e

29. Médium como "arena simbolica che si colloca nella zona de intersezione tra constesti e pratiche discorsive di produzione/ricezione del senso". Rivoltella (2003, 109). El autor recoge explícitamente en este concepto varias ideas: la de espacio, la de mediación y la de interacción. 
interpretaciones, prácticas y concepciones, representaciones y manifestaciones que los usuarios tienen y hacen de estas tecnologías en sus mundos de vida.

Pues bien, esta investigación da un paso adelante en esos aspectos, aportando resultados en varias direcciones:

- $\quad$ en primer lugar, se demuestra la presencia y sentidos del espacio en la utilización de las nuevas tecnologías en función precisamente de los usos o tipo de actividad que ahí se desarrolla;

- $\quad$ en un segundo momento, se identifica y describe la reconfiguración conceptual que conoce el espacio cuando se trata precisamente de entornos virtuales de formación; reconfiguración conceptual, no supresión ni negación del espacio;

- $\quad$ en tercer lugar, se posibilita una lectura pedagógica que lleva a explicar satisfactoriamente los procesos educativos mediados tecnológicamente.

Y todo ello sin hablar de dos espacios diferentes, el espacio real y el virtual, distinción y concepción que en ocasiones dejan traslucir algunos autores -no sólo los entregados a la ciencia ficción- y que resulta perjudicial para un uso adecuado y completo de estas tecnologías por los profesionales de la enseñanza, que se mueven por ello entre el recelo y la desconfianza cuando se habla de formación en espacios virtuales. A la Teoría de la Educación le corresponde "remover obstáculos" que impidan una buena comprensión de los procesos de formación on line; ésa ha sido la pretensión de este artículo.

\section{Límites Del ESTUDiO}

La principal limitación de este estudio deriva del escaso número de sujetos utilizados, aun siendo válidos el diseño metodológico general y el procedimiento estadístico empleado, lo que aconseja replicar la investigación en otros grupos que presenten otros perfiles. Sería una forma de comprobar los resultados, además de obtener información complementaria de indudable interés.

\section{Reconocimientos}

El planteamiento, el desarrollo y los datos aquí utilizados pertenecen a la investigación "Interpretación del espacio en los contextos virtuales de aprendizaje», proyecto financiado por el Ministerio de Educación y Ciencia (España), Dirección General de Investigación, Plan Nacional de I+D+i 2004-2007. Ref. SEJ2005-06517/ EDUC, cuyo investigador principal es el propio autor. Duración: 31 de diciembre de 2005-30 de diciembre de 2008. 
ANÁLISIS DEL ESPACIO EN LOS ENTORNOS VIRTUALES DE FORMACIÓN

\section{BiBLIOGRAFÍA}

Burbules, N. C. (2000) Aporias, webs and passages: Doubt as an Opportunity to Learn, Curriculum Inquiry, 30 (2), 171-187.

- (2002) The Web as a Rhetorical Place, en SNYDer, I. (ed.). Silicon Literacies. London, Routledge, 75-84.

- (2004) Rethinking the virtual (documento digital).

Cairncross, F. (1997) The Death of Distance: How the Communications Revolution will Change our Lives. London, Orion.

Calvani, A. (2001) Educazione, comunicazione e nuovi media, Sfide pedagogiche e cyberspazio. Torino, Utet.

Castells, M. (1996) The Rise of the Network Society. Cambridge, MA, Blackwell.

- (1998) La era de la información. Economía, sociedad y cultura. Vol. 1: La sociedad red. Madrid, Alianza.

CEnter for Research on Information TeChnology and Organizations (CRITO) (1998) Internet Use by Teachers: Conditions of Professional Use and Teacher-Directed Student Use. University of California.

DERRIDA, J. (1999) La deconstrucción en las fronteras de la filosofía. La retirada de la metáfora. Barcelona, Paidós.

Dillenbourg, P. (2000) Virtual Learning Environments, en Eun CONFERENCE 2000. Learning in the New Millennium: Building New Education Strategies for Schools. Bruselas.

Dourish, P. (1999) Where the footprints Lead: Tracking down Other Roles for Social Navigation, en Munro, H.; HöÖK, H. y Benyon, D. (eds.). Social Navigation of Information Space. London, Springer, 15-34.

Echeverría, J. (1999) Los Señores del aire: Telépolis y el Tercer Entorno. Barcelona, Destino.

García del Dujo, Á. (2003a) Pedagogy in virtual learning environments, en Méndez Vilas, A.; Mesa González, J. A. y Mesa González, J. Advances in TechnologyBased Education: Toward a Knowledge-Based Society, volumen I. Badajoz, Junta de Extremadura, 201-210.

- (2003b) Momentos críticos en el uso de las nuevas tecnologías en educación, en IX Congreso Interuniversitario de Teoría de la Educación Calidad, equidad y educación. San Sebastián.

GiBson, W. (1984) Neuromancer. New York, Ace Books.

GidDENS, A. (2003) La constitución de la sociedad. Bases para la teoría de la estructuración. Buenos Aires, Amorrortu.

- (2004) Consecuencias de la modernidad. Madrid, Alianza Editorial.

KolB, D. (2000) Learning places: Building Dwelling Thinking On line, Journal of Philosophy of Education, 34 (1), 121-133.

Lankshear, C.; Peters, M. y Knobel, M. (2000) Information, Knowledge and Learning: Some Issues Facing Epistemology and Education a in a Digital Age, Journal of Philosophy of Education, 34 (1), 17-39.

Mantovani, G. (1995) Comunicazione e identità. Dalle situazioni quotidiani agli ambienti virtuali. Bologna, Il Mulino.

Meyrowitz, J. (1986) No Sense of Place. The Impact of Electronic Media on Social Behavior. New York, Oxford University Press. 
Pearson, M. y Somekh, B. (2003) Concept-Mapping as a Research Tool: A Study of Primary Children's Representations of Information and Communication Technologies, Education and Information Technologies, 8 (1), 5-22.

PéRez López, C. (2005) Métodos estadísticos avanzados con SPSS. Madrid, Paraninfo.

Rivoltella, P. C. (2003) Costruttivismo e pragmatica della comunicazione on line. Trento, Erickson.

Rizvi, F. y Lingard, B. (2000) Globalization and Education: complexities and contingencies, Educational Theory, 50 (4), 419-426.

RoberT, P. (2004) Critique de la dématerialisation, Communication \& Langages, 140, 55-68.

VÁzQuez Gómez, G. (2002) El sistema educativo ante la educación de calidad para todos a lo largo y ancho de la vida, Revista de Educación, número extraordinario, 39-57.

- (2003) Sociedad-red, ciudadanía cognitiva y educación, Revista de Educación, número extraordinario, 13-31.

WALTZ, S. B. (2004) Giving artefacts a voice? Bringing into account technology in educational analysis, Educational Theory, 54 (29), 157-172.

Weston, J. (1994) Old freedoms and new technologies: the evolution of community networking. Canada, University of Waterloo. 